\title{
Uncertainty Analysis of Velocity Measured by Diode-Laser Absorption Spectroscopy in a Scramjet Facility
}

\author{
Fei $\mathrm{Li}^{1}$ and Xilong $\mathrm{Yu}^{2}$ \\ State Key Lab of High Temperature Gas Dynamics, Institute of Mechanics, Chinese \\ Academy of Sciences, Beijing 100190, China \\ Weiwei $\mathrm{Cai}^{3}$ and $\mathrm{Lin} \mathrm{Ma}^{4}$ \\ Department of Aerospace and Ocean Engineering, Virginia Tech \\ Blacksburg, VA 24061, USA
}

\begin{abstract}
This work investigates the error caused by non-uniformities along the line-of-sight in velocity measurement using tunable diode-laser absorption spectroscopy (TDLAS). Past work has demonstrated TDLAS as an attractive diagnostic technique for measuring velocity, which is inferred from the Doppler shift of two absorption features using two crossing laser beams. However, because TDLAS is line-of-sight in nature, the obtained velocity is a spatially-averaged value along the probing laser beams. As a result, non-uniformities in the flow can cause uncertainty in the velocity measurement. Therefore, it is the goal of this work to quantify the uncertainty caused by various non-uniformities typically encountered in practice, including boundary layer effects, the divergence/convergence of the flow, and the methods (direct absorption vs. wavelength modulation) used to fit the Doppler shift. Systematic analyses are performed to quantify the uncertainty under various conditions, and case studies are reported to illustrate the usefulness of such analysis in interpreting experimental data obtained from a scramjet facility. We expect this work to be valuable for the design and optimization of TDLAS-based velocimetry, and also for the quantitative interpretation of the measurements.
\end{abstract}

\section{Introduction}

A mong all the laser diagnostics developed for combustion flows, tunable diode laser absorption spectroscopy (TDLAS) has been demonstrated as an attractive technique offering unique advantages such as fast temporal resolution, quantitative measurements, and low cost. As a result, variations of TDLAS have been developed to monitor multiple flow parameters, including temperature, pressure, velocity, density, and flow rate; and applications of TDLAS have been demonstrated in a wide spectrum of combustion systems ranging from aircraft engine, IC engine, high enthalpy wind tunnel, and supersonic combustion flow. Readers interested in a comprehensive and indepth discussion of the capabilities and applications of TDLAS are referred to a review paper [1].

Despite these unique advantages, the limitation of TDLAS is well recognized: it is a line-of-sight technique in nature and hence its application is limited to flows with negligible non-uniformity. A considerable amount of research efforts have been invested in overcoming this limitation. Past efforts can be broadly divided into two categories. Efforts in the first category analyze the uncertainty caused by non-uniformities, so that measurement uncertainty can be quantified and minimized. For example, after analyzing effects of thermal and concentration boundary layer in concentration and temperature measurements, the uncertainties caused by such boundary layer can be quantified and furthermore optimal wavelengths can be chosen to minimize the uncertainties [2]. The work

${ }^{1}$ Assistant Professor, State Key Lab of High Temperature Gas Dynamics, Institute of Mechanics, Chinese Academy of Sciences, Beijing 100190, China, AIAA Member.

${ }^{2}$ Professor, State Key Lab of High Temperature Gas Dynamics, Institute of Mechanics, Chinese Academy of Sciences, Beijing 100190, China, AIAA Member.

${ }^{3}$ Postdoctoral Research Associate, Department of Aerospace and Ocean Engineering, Blacksburg, Virginia, 24060, AIAA Member.

${ }^{4}$ Associate Professor, Department of Aerospace and Ocean Engineering, Blacksburg, Virginia, 24060, AIAA Associate Fellow. 
presented here fall in this category: we analyzed the effects caused by non-uniformities to quantify uncertainties in velocity measurements and these analyses also suggested ways to minimize the uncertainties. Efforts in the second category attempted to obtain spatial resolution by combining TDLAS with tomography inversion [3-5]. This approach typically requires measurements using multiple probing beams and is not the focus of this study.

In this work, we focus on analyzing the uncertainties of velocity measurements using TDLAS, especially when applied in high speed flows such as those encountered in scramjet facilities [6,7]. Velocity is a critical parameter for propulsion study, and various mechanisms can cause non-uniformity in supersonic flows. Thus, the effects of nonuniformities on velocity measurement merit a careful examination. Recent work includes that performed by Chang et al. $[8,9]$, where the performance of TDLAS-based velocimetry was evaluated in a flow tunnel; and that performed by Brown et al. [10], where the average flow rate in flight test was estimated assuming non-uniform pressure distribution obtained from CFD simulation. These work analyzed the velocity-error caused by boundary layers in non-reacting flow fields, and the influence of non-uniform pressure because of the pressure-induced frequency-shift. These past results were usually application-specific, and therefore this work aims at providing a more general analysis based these past efforts. The approach adopted in this work is to analyze the effects of nonuniformities in general quasi-two-dimensional flows; and the methodology is decouple the non-uniformities into those normal to the overall flow direction (e.g., those caused by boundary layer effects), and those along the overall flow direction (e.g., those caused by flow divergence/convergence or heat transfer). The results obtained from this approach are expected to be applicable to an expanded range of applications.

\section{Mathematical and Physical Background}

The use of TDLAS was first demonstrated by [11] and thorough description of the technique can be found in [12]. A brief summary here is provided to facilitate the discussion in subsequent Sections. Figure 1 illustrates a typical setup of TDLAS-based velocimetry. A laser beam was split to two probing beams, labeled as Beam 1 and Beam 2, respectively in Figure 1. These probing beams cross in the region of interest and define a plane of measurement. Two angels, $\theta_{1}$ and $\theta_{2}$, are then defined in the plane of measurement to specify the direction of the probing beams relative to the flow (assumed to have a uniform velocity distribution). This work defines $\theta_{1}$ and $\theta_{2}$ as the angles between the laser beams and the direction normal to the flow. To facilitate the discussion, the direction of the flow is taken to be the $x$ direction, and direction normal to the flow the $y$ direction.

The wavelength of the laser is modulated to scan an absorption feature of a target species, typically water vapor in combustion flows. The absorption feature measured by the two probing beams, due to their different orientation relative to the direction of the overall flow, will show a frequency shift because of the Doppler effects. The frequency shift is proportional to the velocity of the flow $(V)$ as shown by the following equation:

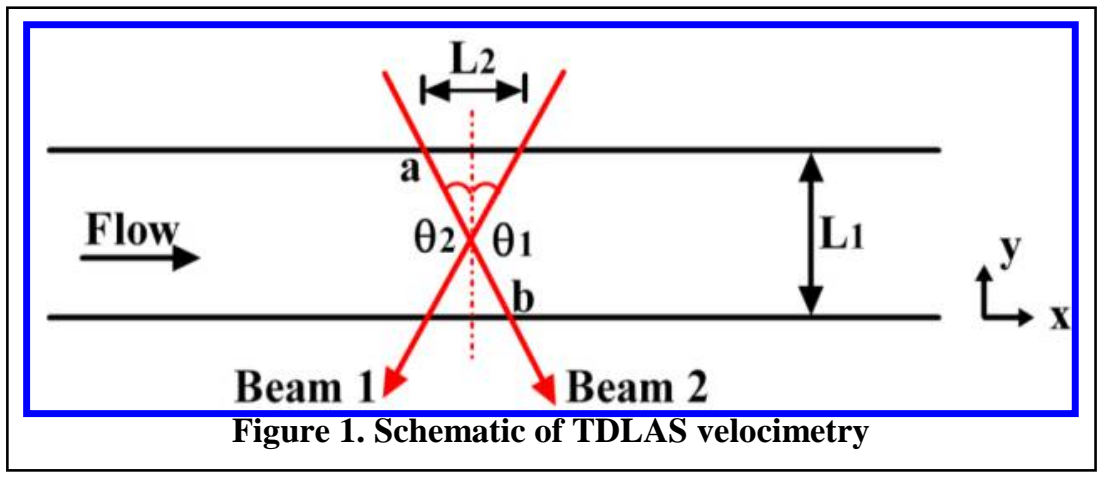

$$
\Delta v_{\text {Doppler }}=\frac{V}{c} v_{0}\left(\sin \theta_{1}+\sin \theta_{2}\right)
$$

Where, $\Delta v_{\text {Doppler }}$ is the Doppler shift between the absorption features measured by the two probing beams, $c$ the speed of light, and $v_{0}$ is the line-center frequency of the absorption line. Practical implementation often sets $\theta_{1}=\theta_{2}$, then Eq. (1) becomes:

$$
\Delta v_{\text {Doppler }}=\frac{2 V}{c} v_{0} \sin \frac{\theta}{2}
$$

where $\theta=\theta_{1}+\theta_{2}$. In the presence of non-uniformity along the path of the probing beams, the velocity measured is a weighted averaged of absorption coefficient $(k)$ in the plane of measurement. The non-uniformity could be in terms of temperature, pressure, and/or velocity. Furthermore, such non-uniformity also causes distortion in the shape 
of absorption feature, leading to uncertainties in determining the absorption peak and eventually in determining the Doppler shift. Analyzing such uncertainties in TDLAS-based velocimetry is the focus of this work.

In our analyses, we decoupled the non-uniformities to provide insights of the uncertainties and to facilitate the application of the results in practice. The non-uniformities are decouple into those along the direction of the flow (i.e., the $x$ direction shown in Figure 1), and those normal to the flow (i.e., the $y$ direction). In practice, nonuniformities in the $y$ direction is typically caused by the existence of boundary layers; and those in the $x$ direction caused by the divergence/convergence of the flow, variations of the boundary layer thickness along the $x$ direction, and/or variations in flow properties due to heat transfer. In this work, uncertainty due to each type of non-uniformity is analyzed in isolation, based on which it is straightforward to analyze multiple types of non-uniformity simultaneously.

As shown in Eqs. (1) and (2), TDLAS-based velocimetry essentially measures the Doppler shift ( $\left.\Delta v_{\text {Doppler }}\right)$ from the absorption signals registered at the two probing beams. And several approaches exist in practice to obtain the Doppler shift. This work analyzes two of them: one based on direct-absorption (DA) and the other based on wavelength-modulation (2f). In the DA approach, the absorption spectra at both probing beams are first calculated, and then $\Delta v_{\text {Doppler }}$ is determined by the frequency difference of an absorption peak on those two spectra. The DA approach is simple when the absorption spectra can be obtained, which requires that a baseline can be obtained [13]. In contrast, the $2 \mathrm{f}$ approach utilizes the peaks in the second harmonic of the signals at both probing beams to determine $\Delta v_{\text {Doppler }}$ [14]. Compared with the DA approach, the $2 \mathrm{f}$ approach can significantly improve the signal-tonoise ratio (SNR) and therefore suitable for applications with weak absorption, and does not require a baseline and therefore applicable under high pressure $[15,16]$. A key parameter in the $2 \mathrm{f}$ approach is the so-called modulation depth, and this work uses a modulation-depth of 2.2 following [17].

\section{Simulation Results}

The analyses were conducted under the context of applications in our scramjet facility as reported in [18], where the typical flow conditions at the measurement location are: Mach number of 1.83 , static temperature of $600 \mathrm{~K}$, and static pressure of $1.0 \mathrm{~atm}$. Water vapor absorption feature centered at $7185.597 \mathrm{~cm}^{-1}$ (i.e., $v_{0}$ ) was used in this analysis. This transition has been applied extensively in practice due to its relatively strong absorption strength at high temperatures and its isolation from interfering transitions in its vicinity $[14,18]$.

Relevant spectroscopic parameters were extracted from HITRAN 2008 [19]. The lower state energy of this absorption line is $E^{\prime \prime}=1045 \mathrm{~cm}^{-1}$. To study the impact of E" on the TDLAS measurement, we assumed two other values of E" $\left(2000 \mathrm{~cm}^{-1}\right.$ and $500 \mathrm{~cm}^{-1}$ ) for this same transition while kept its linestrength and $v_{0}$ fixed. This is a hypothetical study (in practice, each transition has its own set of E", linestrength, and $v_{0}$ ). Nonetheless, the results are valid to illustrate the effects of E"' because the TDLAS measurements (see Eq. 1) do not depend on the absorption strength and depend linearly on $v_{0}$. Therefore, it is straightforward to extend the results obtained in such hypothetical study to practical applications.

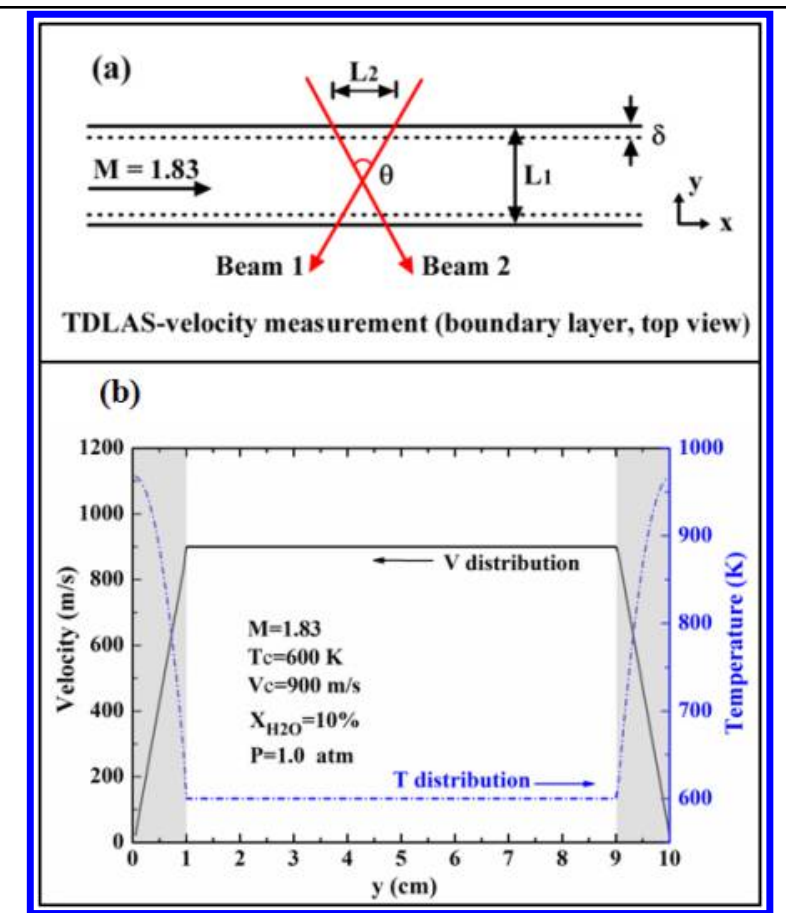

Figure 2. (a) Illustration of TDLAS velocity measurement with boundary layer. (b) Temperature and velocity distributions assumed in the analysis.

Figure 3 shows the uncertainty in velocity measurements due to the non-uniformity in the boundary layers. Here the uncertainty is defined as: 


$$
e=\frac{V_{M}-V_{C}}{V_{C}}
$$

where $V_{M}$ is the velocity that the TDLAS sensor would measure. Two approaches, the DA and $2 \mathrm{f}$ approaches, of obtaining the Doppler shift were compared for transitions with various lower state energies (E"). Note that the error was negative for all these cases (i.e., $V_{M}$ is less than $V_{C}$ ) the flow is slower in the boundary layer than in the core flow.

Figure 3 was generated for conditions representing a typical flow condition corresponding to the "starting point" of scramjet engine in fly tests [20]. More specifically, the main stream flow velocity is $V_{C}=900 \mathrm{~m} / \mathrm{s}$, the temperature was taken to be constant $\left(T_{C}=600 \mathrm{~K}\right)$ in the core flow, and the temperature distribution in the boundary layers was calculated under the isentropic assumption from the velocity distribution as shown in Figure 2(b), the static pressure was assumed to be uniform at $1 \mathrm{~atm}(P=1.0 \mathrm{~atm})$, the Mach number (Ma) was 1.83 in the core flow, and the mole fraction of water vapor was assumed to be uniform at $10 \%(X=10 \%)$.

The results shown in Figure 3 provide several useful observations. First, obviously, the error caused by boundary layer increases with the thickness of the boundary relative to width of the core flow (quantified by $\delta / L_{l}$ in Figure 3). Second, the errors are smaller when the $2 \mathrm{f}$ method was used than when the DA method was used to determine the Doppler shift. Our explanation to the superiority of the $2 \mathrm{f}$ method here is that the $2 \mathrm{f}$ method is less sensitive to the distortion in the shape of the absorption feature.

Non-uniformities along the $x$ direction also occur in supersonic flows due to a variety of reasons. A shockwave intercepting the probing beams represents an extreme case of non-uniformity along the $x$ direction. Here, we analyze effects caused by other factors including the divergence/convergence of the flow, variations of the boundary layer thickness, and/or variations in flow properties due to heat release. We consider the effects caused by the convergence/divergence of the flow, as schematically shown in Figure 4, where a TDLAS-based velocimetry is applied to measure the velocity in the section with a diverging angle of $10^{\circ}\left(\varphi=10^{\circ}\right)$.. In this case, the flow properties vary along the $x$ direction and the variations were modeled by assuming an isentropic flow. Figure 6 shows the error in velocity measurements caused by

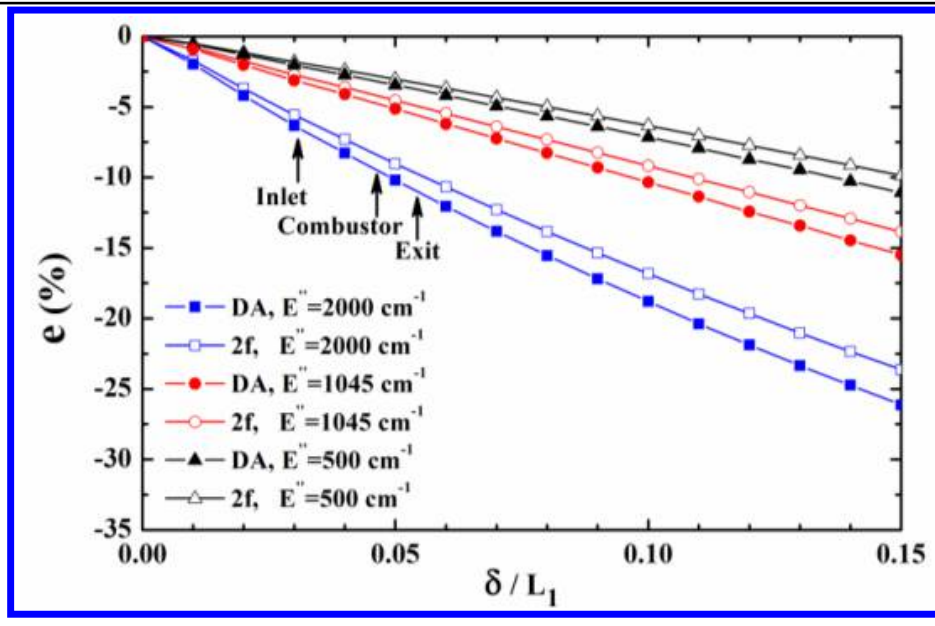

Figure 3. Velocity error caused by non-uniformity in the boundary layers.

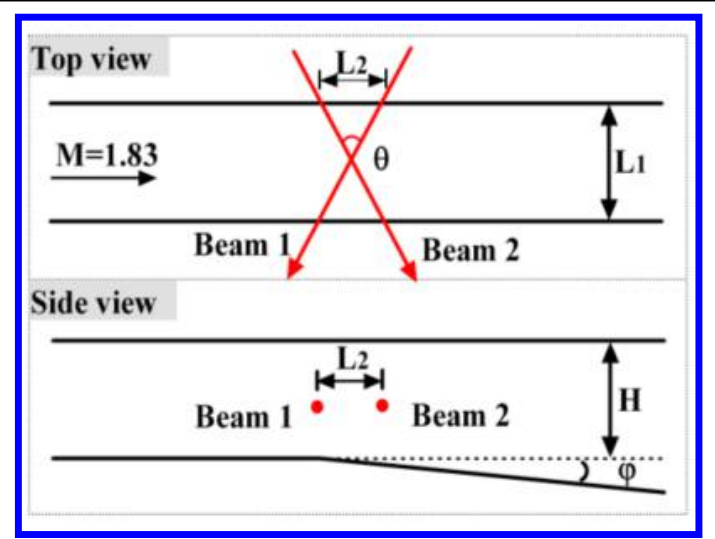

Figure 4. Velocity error caused by non-uniformity in the boundary layers.

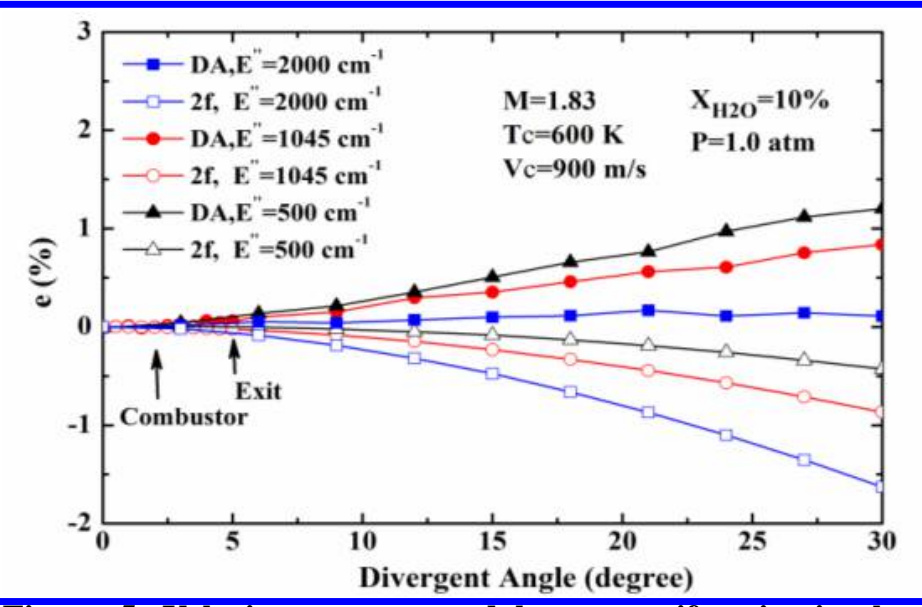

Figure 5. Velocity error caused by non-uniformity in the boundary layers.

4

American Institute of Aeronautics and Astronautics 
such non-uniformities. Here, the error is defined as:

$$
e=\frac{V_{M}-V_{a v g}}{V_{a v g}}
$$

where $V_{\text {avg }}$ is the arithmetically averaged velocity along $L_{2}$. As shown in Figure 6, velocity error is generally within $\pm 1 \%$ when the divergence angle is less than $20^{\circ}$. For practical scramjet applications, divergence angle in the combustor and exit is normally less than $5^{\circ}$ as indicated on Figure 6, which results in a velocity error less than $0.2 \%$ for the conditions shown here. Two interesting observations can be made from these results. First, such error is significantly smaller than that caused by non-uniformity in the $y$ direction as discussed previously for typical scramjet flows; and second, such error is also significantly smaller than the variation of $V$ itself in the $x$ direction (in the case shown here, $V$ itself varies for about $7 \%$ along $L_{2}$ ). Our explanation for these two observations is that the probing beams cross the flow at an angle and measure a velocity that is averaged along $\mathrm{L}_{2}$. Even though this average is not an arithmetic average, it is very close the arithmetic average. The following results will show that both observations remain valid for more general cases where non-uniformities exist in multiple parameters along the $x$ direction.

In practice, other factors can cause nonuniformities along the $x$ direction in addition to the divergence/convergence of the flow. For example, in scramjet combustors, the static temperature and velocity along the $x$ direction may vary because of heat release or the increasing thickness of the boundary layers. In this work, we analyzed a simple case where the static temperature $(T)$, pressure $(P)$, and velocity $(V)$ all vary along the $x$ direction in a linear fashion, as shown in Figure 6. The rate of variation for the static temperature, pressure, and velocity are $k_{T}, k_{P}$, and $k_{V}$, respectively. Such a linear variation represents a good approximation for the conditions in our scramjet facility; and the variations (represented by $\Delta T, \Delta P$, and $\Delta V$ in Figure 6) along $L_{2}$ typically does not exceed $20 \%$ of the incoming conditions at $x=0$ (represented by $T_{0}, P_{0}$, and $V_{0}$ in Figure $6)$.

The error caused by such simultaneous variation in $T, P$, and $V$ along the $\mathrm{x}$ direction was simulated, and Figure 7 shows the maximum absolute error (i.e., $\max |e|)$ when $T, P$, and $V$ all vary within $\pm 20 \%$ of their

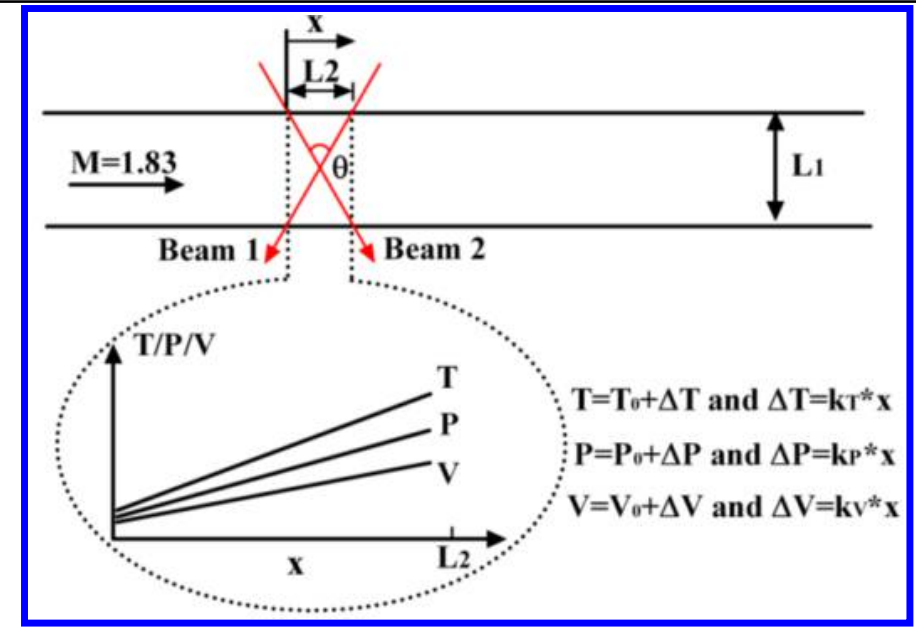

Figure 6. Illustration of TDLAS velocity measurement with non-uniformity simultaneously present in temperature, pressure, and velocity along $\mathbf{L}_{2}$.

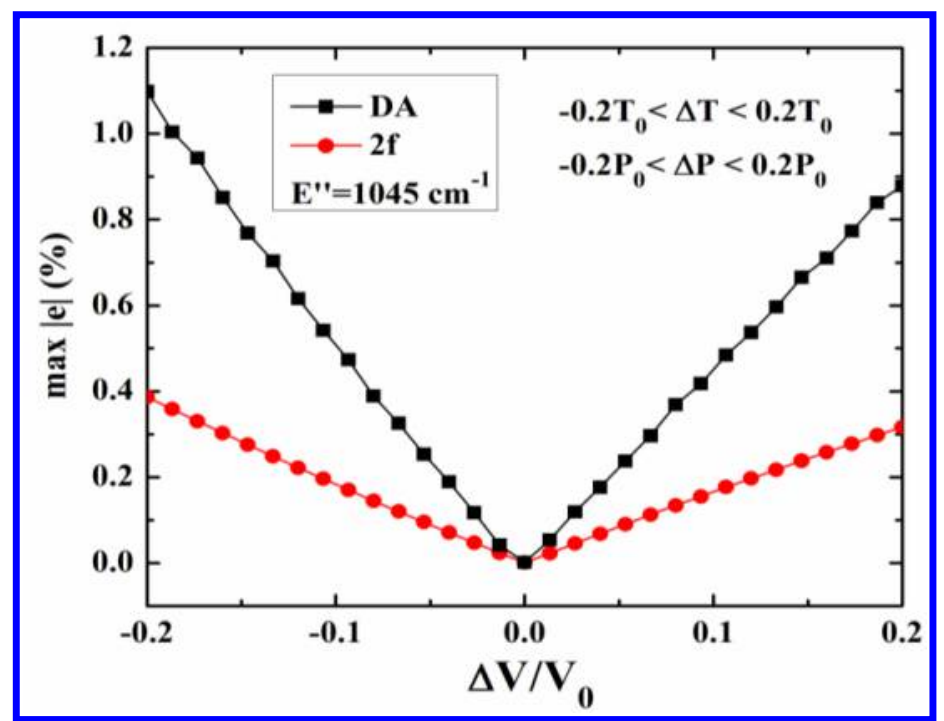

Figure 7. Maximum velocity measurement error versus $k_{\mathrm{v}}$.

incoming values. As shown, the maximum error of velocity is less than $1.2 \%$, indicating that even the flow parameter varies substantially along $\mathrm{L}_{2}$ (normally smaller than $5 \mathrm{~cm}$ ), the velocity error remains less than $1 \%$ relative to the arithmetic average if a suitable absorption line is used. 


\section{Analysis of Experimental Data}

This section applies the analysis presented above to experimental data obtained in our scramjet facility. The scramjet facility is a direct-connected facility capable of simulating supersonic combustion under flight condition. Experiments were performed in a two-dimensional combustor fueled with ethylene, with a test section of the size of $40 \times 85 \mathrm{~mm}^{2}$. The detailed description of this facility and the experiments can be found in [21]. Five representative cases were examined and the results summarized in Figure 8. These five cases include velocity measured at the inlet (Case 1) and the exit (Case 2) of the scramjet combustor at Mach 1.8, and velocity measured at the inlet (Case 3), the combustor (Case 4), and the exit (Case 5) of the scramjet at Mach 2.5. Figure 8 shows the relative error in velocity for each case.

These error analyses were performed using the method demonstrated by Figures 3, 5, and 7 . Here we use Case 4 as an example to elucidate the steps of our analyses. First, the thickness of the boundary layer $(\delta)$ is estimated to be $4 \mathrm{~mm}$ for the combustor of our directconnected scramjet facility according to CFD simulations [22]. The measurement pathlength $\left(L_{l}\right)$ was 85 $\mathrm{mm}$. Therefore from Figure 3, the error caused by the boundary layer in this case is estimated to be $4.8 \%$ (the DA method was used with E" $=1045$ $\mathrm{cm}^{-1}$ in this measurement). Second, the divergence angle in the experiments was $2^{0}$. Then from Figure 5 , the error caused by the

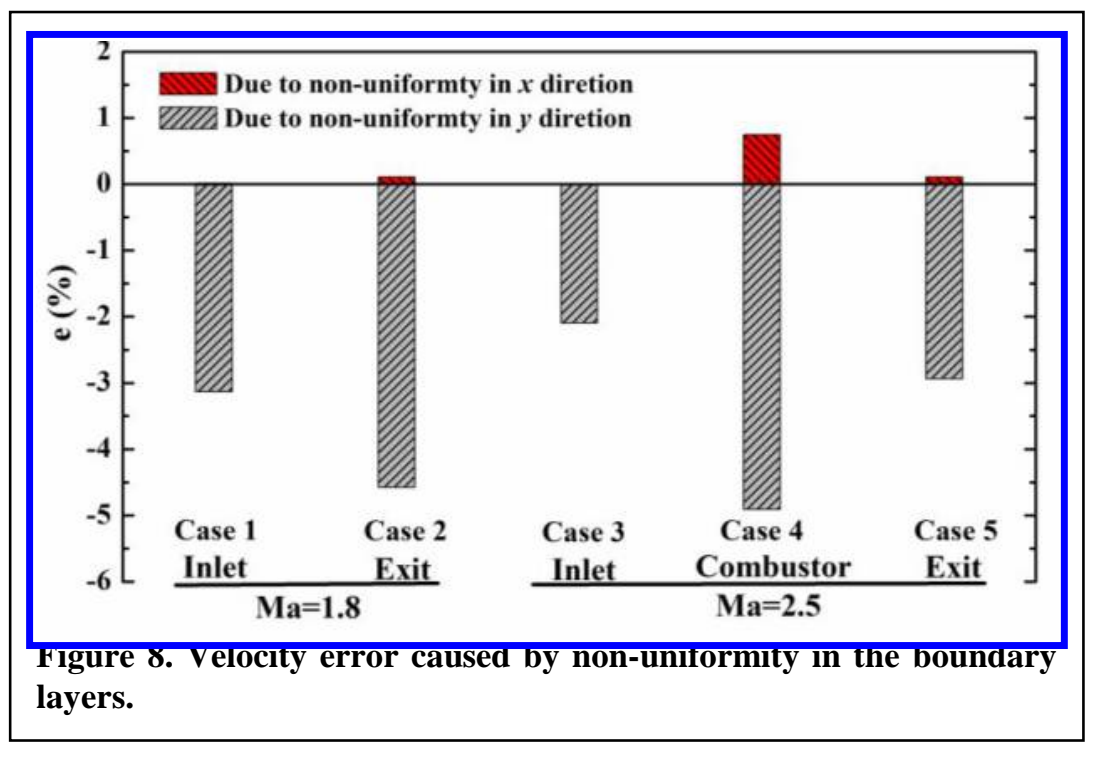
divergence angle in this case was estimated to be $0.1 \%$. Third, along the $\mathrm{L}_{2}$ direction, the variation of the parameters in the combustor was estimated to be: $\Delta T<0.1 \times T_{0}, \Delta P<0.2 \times P_{0}, \Delta V<0.05 \times V_{0}$. Therefore, using the results shown in Figures 7 , velocity error caused by non-uniformities along the $\mathrm{L}_{2}$ direction was determined to be $1 \%$.

From these results, it can be concluded that that the error in scramjet flows is mainly due to the non-uniformities in the boundary layers. It should be emphasized that the flow fields were assumed to be quasi-2D in this work, i.e., the flow parameters along $\mathrm{L}_{1}$ are uniform except in the boundary layers. When significant 3D effects exist (e.g., when a shock wave intercepts the probe beams), both the overall error and the relative contribution due to $x$ - and $y$ direction non-uniformities will be different than those reported here, and the interpretation of the TDLAS measurements is more complicated [10].

\section{Conclusion}

This work investigated the uncertainty in velocity measurements using TDLAS-based techniques under the context of scramjet flows. This study focuses on quantifying the uncertainties caused by non-uniformities in the flow. The non-uniformities typically encountered in practice were decoupled into those in the direction normal to the mean flow (i.e., boundary layer effects the $y$ direction) and those in the direction along the mean flow (the $x$ direction). Two variations of TDLAS, the direct-absorption and wavelength-modulation, were examined.

Two main findings can be summarized from the results obtained in this study. First, under typical conditions in scramjet flows, the error caused by the non-uniformities in $x$ direction is significantly smaller than that caused by the boundary layer effects and also than the variation of the velocity itself in the $x$ direction. Second, the $2 \mathrm{f}$ method is less sensitive to the boundary layer effects than the DA method in determining the Doppler shift. The boundary layers effects distort the absorption line shape to deviate from the Voigt profile. Unlike the DA method, the $2 \mathrm{f}$ method does not rely on the assumption of the Voigt profile to determine the location of the absorption peak. It detected the peak by analyzing the derivative of the absorption shape and is less sensitive to the distortion of the absorption shape. As a result, in our results, the $2 \mathrm{f}$ method yielded more accurate Doppler shift than the DA method. 
These findings were then applied to analyze the experimental data obtained in a scramjet facility, illustrating the usefulness of the results reported. We expect this work to be valuable for quantifying the experimental data, the data analysis, and also the design and optimization of TDLAS-based velocimetry for high speed flows.

\section{References}

1. R. K. Hanson, "Applications of quantitative laser sensors to kinetics, propulsion and practical energy systems," Proceedings of the Combustion Institute 33, 1-40 (2011).

2. O. Y. Xiang, and P. L. Varghese, "Line-of-Sight Absorption-Measurements of High-Temperature Gases with Thermal and Concentration Boundary-Layers," Applied Optics 28, 3979-3984 (1989).

3.W. W. Cai, D. J. Ewing, and L. Ma, "Application of simulated annealing for multispectral tomography," Computer Physics Communications 179, 250-255 (2008).

4.J. W. S. T. Sanders, J. B. Jeffries, and R. K. Hanson "Diode-laser absorption sensor for line-of-sight gas temperature distributions " Appl. Opt 40, 4404-4415 (2001).

-5. X. Liu, J. B. Jeffries, and R. K. Hanson, "Measurement of nonuniform temperature distributions using line-ofsight absorption spectroscopy," Aiaa Journal 45, 411-419 (2007).

6. A. D. Griffiths, and A. F. P. Houwing, "Diode laser absorption spectroscopy of water vapor in a scramjet combustor," Applied Optics 44, 6653-6659 (2005).

7. G. B. Rieker, J. B. Jeffries, R. K. Hanson, T. Mathur, M. R. Gruber, and C. D. Carter, "Diode laser-based detection of combustor instabilities with application to a scramjet engine," Proceedings of the Combustion Institute 32, 831-838 (2009).

8. C. L. S. Leyen S. Chang, Jay B. Jeffries, and Ronald K. Hanson, "Supersonic mass flux measurements via tunable diode laser absorption and non-uniform flow modeling," 49th AIAA Aerospace Sciences Meeting including the New Horizons Forum and Aerospace Exposition 4 - 7 January 2011, Orlando, Florida, AIAA 2011-1093 (2011).

9. L. S. Chang, J. B. Jeffries, and R. K. Hanson, "Mass Flux Sensing via Tunable Diode Laser Absorption of Water Vapor," Aiaa Journal 48, 2687-2693 (2010).

10. M. B. a. T. Barhorst, 17th AIAA International Space Planes and Hypersonic Systems and Technologies Conference San Francisco, California, Apr. 11-14, 2011, AIAA-2011-2359 (Post-Flight Analysis of the DiodeLaser-Based Mass Capture Experiment Onboard HIFiRE Flight 1).

11. L. C. Philippe, and R. K. Hanson, "Laser-Diode Wavelength-Modulation Spectroscopy for Simultaneous Measurement of Temperature, Pressure, and Velocity in Shock-Heated Oxygen Flows," Applied Optics 32, 60906103 (1993).

12. M. P. Arroyo, S. Langlois, and R. K. Hanson, "Diode-laser absorption technique for simultaneous measurements of multiple gasdynamic parameters in high-speed flows containing water vapor," Appl. Opt. 33, 3296-3307 (1994).

13. J. A. Silver, and D. J. Kane, "Diode laser measurements of concentration and temperature in microgravity combustion," Measurement Science \& Technology 10, 845-852 (1999).

14. J. T. C. Liu, G. B. Rieker, J. B. Jeffries, M. R. Gruber, C. D. Carter, T. Mathur, and R. K. Hanson, "Nearinfrared diode laser absorption diagnostic for temperature and water vapor in a scramjet combustor," Applied Optics 44, 6701-6711 (2005). 
15. H. J. Li, G. B. Rieker, X. Liu, J. B. Jeffries, and R. K. Hanson, "Extension of wavelength-modulation spectroscopy to large modulation depth for diode laser absorption measurements in high-pressure gases," Applied Optics 45, 1052-1061 (2006).

16. G. B. Rieker, J. B. Jeffries, and R. K. Hanson, "Calibration-free wavelength-modulation spectroscopy for measurements of gas temperature and concentration in harsh environments," Applied Optics 48, 5546-5560 (2009).

17. X. Zhou, J. B. Jeffries, and R. K. Hanson, "Development of a fast temperature sensor for combustion gases using a single tunable diode laser," Applied Physics B-Lasers and Optics 81, 711-722 (2005).

18. X. Y. Fei Li, Hongbin Gu,Zhi Li,Lihong Chen, and Xinyu Chang "Measurement of temperature, velocity and water vapor concentration in a scramjet combustor based on near-infrared diode laser absorption " in Proceedings of Seventeenth AIAA International Space Planes and Hypersonic Systems and Technologies AIAA 2011-2214, (American Institute of Aeronautics and Astronautics 2011) (2011).

19. L. S. Rothman, I. Gordon, A. Barbe, D. C. Benner, P. Bernath, M. Birk, V. Boudon, L. Brown, A. Campargue, and J. P. Champion, "The HITRAN 2008 molecular spectroscopic database," Journal of Quantitative Spectroscopy and Radiative Transfer 110, 533-572 (2009).

20. a. D. R. W. kristen N.Roberts, "Analysis and Design of a Hypersonic Scramjet Engine with a Transition Mach Number of 4.0," 47th AIAA Aerospace Sciences Meeting Including The New Horizons Forum and Aerospace Exposition AIAA 2009-1255 (2009).

21. X. Y. Fei Li, Hongbin Gu, Zhi Li, Yan Zhao, Lin Ma, Lihong Chen, Xinyu Chang, "Simultaneous Measurements of Multiple Flow Parameters for Scramjet Characterization Using Tunable Diode-Laser Sensors," Applied Optics 50, 6697-6707 (2011).

22. L. C. T. Wan, J.Wang, X.Fan, and X.Chang, "Computational study of supersonic combustion using three kerosene reaction mechanism models," Proceedings of the 13th Asian Congress of Fluid Mechanics 17-21 December 2010, Dhaka, Bangladesh (2010). 